\title{
Continuous Control and Synchronization in Chaotic Systems
}

\author{
T. KAPITANIAK \\ Division of Control and Dynamics, Technical University of Lodz \\ Stefanowskiego 1/15, 90-924 Lodz, Poland.
}

\begin{abstract}
We show that two identical chaotic systems can be synchronized by applying the method of continuous chaos control. The developed method is especially useful for higher-dimensional systems. We also discuss the possible applications of our synchronization scheme in geophysical systems.
\end{abstract}

\section{INTRODUCTION}

Synchronization of periodic oscillations in coupled systems has been known since the time of Huygens [1]. In early 17 th century he was probably the first scientist who observed that a couple of pendulum clocks hanging on a light beam had synchronized, i.e., the pendulums oscillate with equal frequencies and opposite phases.

The essential property of a chaotic trajectory is that it is not asymptotically stable. Closely correlated initial conditions have trajectories which quickly become uncorrelated. Despite this obvious disadvantage, it has been established that synchronization of two chaotic systems (the complete coincidence of oscillations forms) is possible [2-4] and has potential practical applications in secure communications [8,9]. Pecora and Carroll showed [2] that there exists the class of chaotic systems for which synchronization can be achieved. Consider a system that can be divided into the drive subsystem (whose largest Lyapunov exponent is positive) and the driven subsystem (with all negative Lyapunov exponents). In this case trajectories from two identical driven subsystems can be synchronized if the same driven system is used. This result has been numerically and experimentally verified mainly on electrical systems [2-4]. De Sousa Vieira, Lichtenberg and Lieberman [3] showed that the boundary of possible synchronization and nonsynchronization is strictly connected with the transition from chaotic to hyperchaotic behaviour that is characterized by at least two positive Lyapunov exponents $[13,14]$.

Recently chaos controlling method of Ott, Grebogi and Yorke (OGY) [10] has been used to synchronize chaotic systems [7]. It was shown that by applying small, judiciously chosen, temporal-parameter perturbation to one of the chaotic system we can stabilize its orbit around a chaotic trajectory of the other 
system achieving synchronization of the two systems. The OGY method has been applied to control chaotic orbits by Mehta and Henderson [5]. Although this idea can be directly applied to synchronization problems it may not be easily used for dynamical systems more general than one considered in [5].

The OGY method requires a permanent computer monitoring of the state of the system and deals with Poincare map to evaluate the changes of the parameter. Since the corrections of the control parameter are rare and small, the fluctuation noise leads to occasional bursts of the system into the region far from the desired orbit causing breaks in control or synchronization. The frequency and duration of these breaks increase with the increase of noise intensity. To avoid these problems Pyragas [11] proposed the method of a timecontinuous control. This method is based on the construction of a special form of time-continuous perturbation, and feedback mechanism. One of the proposed feedback controlling loops in which the external periodic perturbation is used, is shown in Fig. 1. In [11,12] it was shown that if the external periodic perturbation is in the form of unstable periodic orbit (UPO) embedded in the chaotic attractor under certain conditions UPO can be stabilized. In this paper we discuss other properties of the slightly modified continuous controlling loop of Fig. 1.

The plan of this paper is as follows. In Sec. 2 we discuss the possibility of applying a continuous chaos controlling scheme [11] to achieve a synchronization of two chaotic systems. The method we developed is a generalization of the method of controlling dependence on initial conditions [6]. Section 3 presents possible applications of our synchronization scheme in geophysical models. Finally we summarize our results in Sec. 4

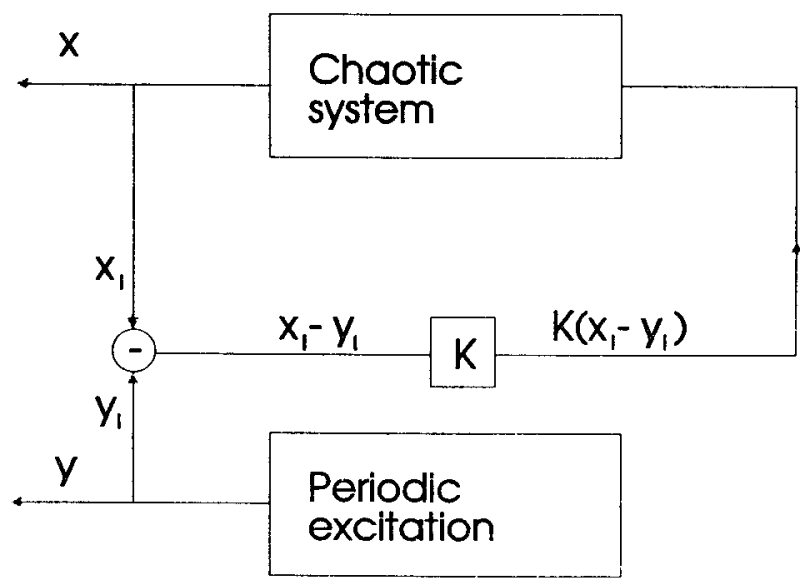

Fig. 1. Scheme of continuous chaos control method. 


\section{SYNCHRONIZATION BY CONTINUOUS CONTROL}

To synchronize two chaotic systems,

$$
\begin{aligned}
& \dot{x}=f(x) \\
& \dot{y}=f(y)
\end{aligned}
$$

where $\mathbf{x}, \mathbf{y} \in \mathbf{R}^{\mathbf{n}}$, that we call $\mathrm{A}$ and $\mathrm{B}$, we use the strategy which is schematically illustrated in Fig. 2.

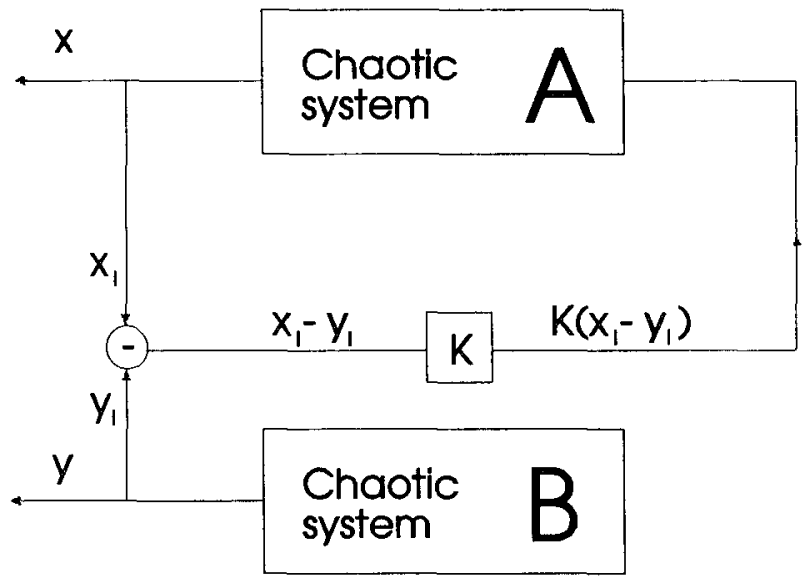

Fig. 2. Scheme of our method of synchronization of two chaotic systems.

We assumed that some state variables of both systems A and B can be measured. Let's say that we can measure signal $x_{i}(t)$ from the system $A$ and signal $y_{i}(t)$ from $B(i=1,2, \ldots, n)$. Chaotic systems $A$ and $B$ are coupled unidirectionally in such a way that the difference $D(t)$ between the signals $x_{i}(t)$ and $y_{i}(t)$ is used as a control signal

$$
F(t)=K\left[x_{i}(t)-y_{i}(t)\right]=K D(t)
$$

introduced into one of the chaotic systems ( $\mathrm{A}$ in Fig. 2) as a negative feedback. $\mathrm{K}>0$ is an experimentally adjustable weight of the perturbation and we discuss its selection later. An experimental realization of such a feedback presents no difficulties for many practical systems. The important feature of the perturbation signal (2) is that it does not change the solution of equation (1B). When the synchronization is achieved $F(t)$ becomes zero so the chaotic systems $A$ and $B$ become practically uncoupled.

We illustrate our synchronization procedure on the example of two identical Duffing's equations

$$
\begin{aligned}
& \ddot{x}+a \dot{x}+x^{3}=B \cos t \\
& \ddot{y}+a \dot{x}+x^{3}=B \cos t
\end{aligned}
$$


It is well-known that for $\mathrm{a}=0.1, \mathrm{~B}=10.0$ equations (3) show chaotic behaviour [15] and if $\mathbf{x}(0)$ is slightly different than $y(0)\left(x=[x, x, 1]^{T}, y=[y, y, 1]^{T}\right)$ trajectories $x(t)$ and $y(t)$ diverge exponentially from each other. To achieve synchronization we add perturbation signal (2) to equation (3A) obtaining a coupled system

$$
\begin{aligned}
& \ddot{x}+a \dot{x}+x^{3}=K(y-x)+B \cos t \\
& \ddot{y}+a \dot{y}+y^{3}=B \cos t
\end{aligned}
$$

In our numerical experiment we have studied the dependence of the synchronization time $t_{s}$, defined as the time taken to reach the synchronization with the assumed precision $10^{-4}$, i.e. $|y(t)-x(t)|<10^{-4}$. For $\mathrm{K}$ in the interval $(0.01,0.1)$ we estimated $t_{s}$ for 1000 randomly chosen initial conditions and averaged them. Our results are shown in Fig. 3 (dots) where as expected, $t_{s}$ decreases when $\mathrm{K}$ increases. To verify the effectiveness of our method in noisy situation we add white noise to one of the chaotic systems - B. We found that synchronization is still possible and that the noise has no influence on the synchronization time $t_{s}$ as shown in Fig. 3 (squares).

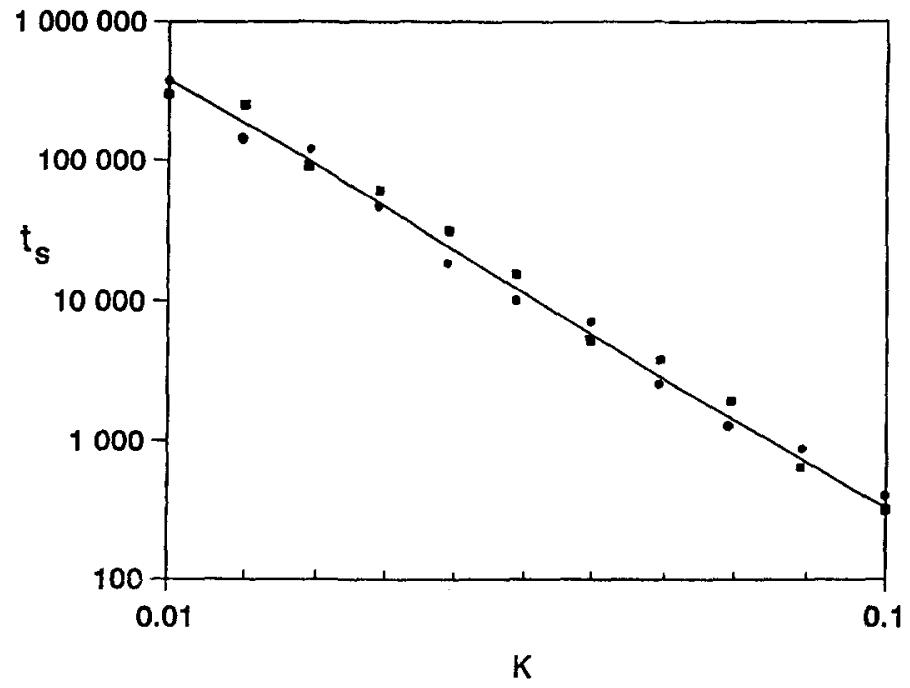

Fig. 3.3. Averaged time to achieve synchronization $\left\langle\mathrm{t}_{\mathrm{s}}\right\rangle$ vs. coupling stiffness $\mathrm{K}$;

without noise, $\mathbf{E}^{-1}$ white noise with amplitude $10^{-3}$ added to the chaotic system $B$.

The presented example showed that our method is a very convenient way to synchronize multidimensional systems by feeding back a single variable. Generally it has to be one of the state variables described by a drive subsystems (in the classification of Pecora and Carroll [2]) of chaotic systems A and B as feeding back variables from driven subsystem gives no results in continuous chaos control method [12]. However due to the limitations of continuous chaos control methods [12] feeding back one state variable it is not always successful. One can easily show that synchronization can be achieved only if the number of positive Lyapunov exponents of the coupled system equals to the number of positive Lyapunov exponents of a single system. In our example only one positive Lyapunov exponent in the spectrum of equations (4) is allowed to achieve synchronization. Knowing equations of chaotic systems A and B we can easily check the 
above condition by direct computation of Lyapunov exponents. If the systems A and B are given only by time series we can check properties of correlation dimension ( one scaling region for chaotic attractor and at least two scaling regions for hyperchaos) [14]. If Lyapunov exponents condition is fulfilled the coupled system (4) evolves on the same manifold on which both chaotic systems evolve and that is why synchronization can be obtained. When it is not fulfilled the coupled system evolves on higher-dimensional manifold on which hyperchaotic attractor exists and according to De Sousa Vieira, Lichtenberg and Lieberman [3] synchronization cannot be obtained. As shown in [15] by weakly coupling two chaotic systems, as in our synchronization procedure it is not so easy to find hyperchaos. But if we are unlucky to find more positive Lyapunov exponents for the coupled system than we have in the original systems we can try to avoid hyperchaos either by changing coupling stiffness $\mathrm{K}$ or by simultaneously feeding back more state variables of chaotic systems $\mathrm{A}$ and $\mathrm{B}$. Coupling stiffness $\mathrm{K}$ has to be chosen in such a way to achieve small synchronization time $\mathrm{t}_{\mathrm{h}}$, but it cannot be too high to avoid hyperchaos. It is hopefully expected that by feeding back small number of variables one can be able to synchronize chaotic systems with more dimensions.

\section{APPLICATIONS IN GEOPHYSICAL SYSTEMS}

The difficulty of carrying out long-term predictions of atmospheric dynamics and the evolution of climate is a problem of obvious concern. Nowadays there is increasing awareness that deterministic chaos might provide a natural prototype for complexity of atmospheric and climatic dynamics. Periodicity is the first apparent characteristic of the behaviour of many geophysical fluid dynamic systems, but atmospheric and oceanic flows often exhibit substantial coherent features, localized in either or both of space and time, which occur sporadically and unpredictably but with a certain statistical regularity also called extended-range atmospheric prediction. Such features are exemplified by blocking patterns in the mid-latitude atmosphere, or by persistent anomalies (of which El Nino is the spectacular example [16]).

In this section we propose the possible mechanism of extended-range atmospheric prediction based on the continuous chaos control scheme of Fig. 1. As the chaotic system we consider Lorenz model [17]

$$
\begin{gathered}
\dot{X}=-\sigma X+\sigma Y \\
\dot{Y}=-X Z+r X-Y \\
\dot{Z}=X Y-b Z
\end{gathered}
$$

which can be taken as a paradigm for the chaotic extratropic atmosphere [18]. $\mathrm{X}, \mathrm{Y}$ and $\mathrm{Z}$ can be considered as representing Rossby wave components of the extratropical general circulation [18]. Eq. (5) is coupled with the linear oscillator

$$
\begin{gathered}
\dot{W}=-\Omega V-k\left(W-W^{*}\right) \\
\dot{V}=\Omega\left(W-W^{*}\right)-k V
\end{gathered}
$$


which represents the tropical atmosphere [18]. Here, $\Omega$ is taken to be the frequency of the dominant internal mode of large-scale variability of the tropics, the Madden-Julian oscillations ( $=1.5$ in nondimensional time) [19]. $\mathrm{V}$ and $\mathrm{W}$ represent two phase-quadrature components of the tropical oscillations. For example $\mathrm{W}$ can be considered as representing the Walker circulation [18].

Application of continuous chaos control method of Fig. 1 requires consideration of the following coupled equations:

$$
\begin{gathered}
\dot{X}=-\sigma X+\sigma Y \\
\dot{Y}=-X Z+r X-Y+K(W-Y) \\
\dot{Z}=X Y-b Z \\
\dot{W}=-\Omega V-k\left(W-W^{*}\right) \\
\dot{V}=\Omega\left(W-W^{*}\right)-k V
\end{gathered}
$$

The examples of numerical calculations for $\sigma=10.0, \mathrm{r}=28.0, \mathrm{~b}=8 / 3, \Omega=1.5, \mathrm{k}=0.1$ and $\mathrm{W}^{\prime \prime}=0$ are shown in Fig. 4(a,b). In Fig. 4(a) we showed the original attractor of Lorenz system given by eq. (5) (or

(a)

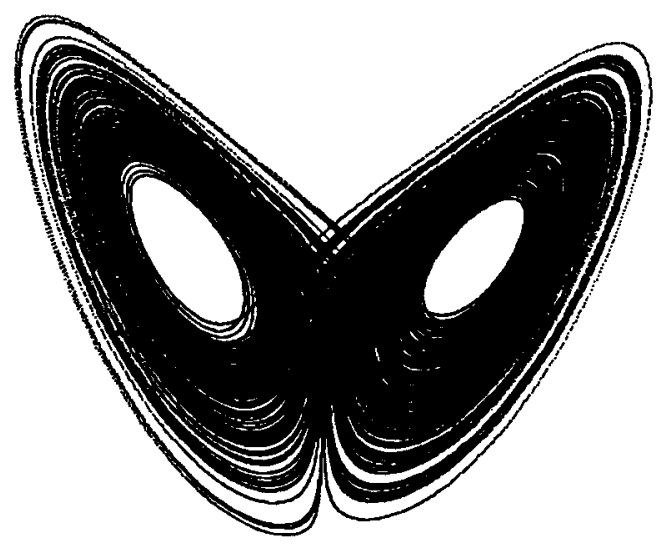

(b)

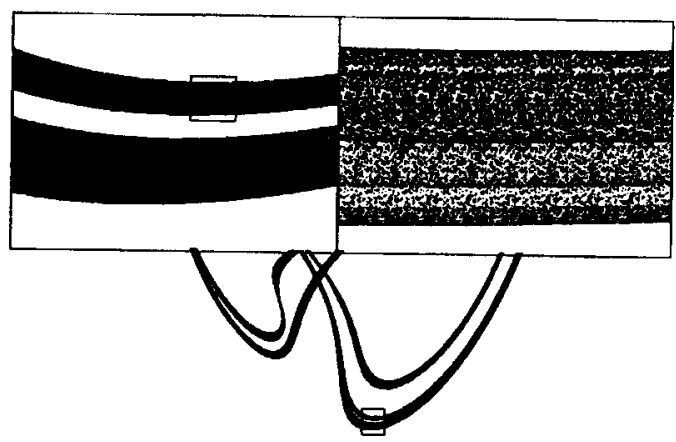

Fig 4. Evolution of Lorenz system

a) unmodified system eq.(5): $\sigma=10.0, r=28.0, b=8 / 3$,

b) controlled system eq. (7): $\sigma=10.0, r=28.0, b=8 / 3, \Omega=1.5, k=0.1, W^{*}=0, K=5.0$. 
eq. (7) with $\mathrm{K}=0$ ) while in Fig. 4(b) the same attractor modified by coupling of eq. (5) with eq.(6) (eq. (7) with $\mathrm{K}=5.0$ ) is presented. In this case application of continuous control scheme did not result in obtaining periodic behaviour as the external periodic perturbation was not taken in the form of unstable periodic orbit of original chaotic system. On the other hand although the attractor of Fig. 4(b) is chaotic its dimension is smaller than the dimension of original chaotic attractor of Fig. 4(a). The dynamics of the modified system (7) is far more predictable than dynamics of the system (5).

The proposed coupling can be one of the possible explanations of the physical mechanism of the extended-range atmospheric prediction observed in geophysical systems. The complete study of system (7) and its relation to real geophysical data will be given elsewhere [20].

\section{CONCLUSIONS}

We presented the method for achieving synchronization of two chaotic systems by applying a continuous chaos control scheme. Our method does not require a monitoring of chaotic trajectories or application of targeting procedure that was necessary in the previous method [4]. Due to the continuity of the control, our synchronization method is noise resistant and can be easily applied to the experimental systems, especially to secure communication systems [8,9].

We also pointed out possible applications of continuous control scheme in geophysical systems, especially to explain the physical mechanism of extended-range atmospheric prediction.

Acknowledgement - This work has been supported by KBN (Poland) under project no: 333579102.

\section{REFERENCES}

1. C. Hugenii, Horoloqium oscilatorium, Paris: (1673).

2. L.M. Pecora and T.L. Carroll, Phys. Rev. Lett. 64, 821 (1990); IEEE Trans. Circuits Syst. 38, 453 (1991); Phys. Rev. A44, 2374 (1991)

3. M. de Sousa Vieira, A.J. Lichtenberg, and M.A. Lieberman, Phys. Rev. A46, 7359 (1992)

4. P. Landa, M. Rosenblum, Appl. Mech. Rev., 46, 416 (1993)

5. M.J. Mehta and R.M. Henderson, Phys. Rev. A44, 4861 (1991)

6. T. Kapitaniak, In: Future Directions of Nonlinear Science in Physical and Biological Systems;

P.L. Christiansen (ed.), pp.115-118, Plenum, New York 1993.

7. Y.-Ch. Lai and C. Grebogi, Phys. Rev. 47E, 2357 (1993)

8. U. Parlitz, L.O. Chua, Lj. Kocarev, K. Halle, and A. Shang, Int. J. Bif. Chaos 2, 973 (1992)

Lj. Kocarev, K. Halle, K. Eckert, and L.O. Chua, Int. J. Bif. Chaos 2709 (1992)

9. K.M. Cuomo and A.V. Oppenheim, Phys. Rev. Lett. 71, 65 (1993)

10. E. Ott, C. Grebogi and Y.A. Yorke, Phys. Rev, Lett. 64, 1196 (1990).

11. K. Pyragas, Phys. Lett. A170, 421 (1992) 
12. Z. Qu, G. Hu and B. Ma, Phys. Lett. A178, 265 (1993)

13. O. Rossler, Phys. Lett. A 71, 155 (1986)

T. Kapitaniak and W.-H. Steeb, Phys. Lett. 152A, 33 (1991)

14. T. Kapitaniak, Phys. Rev. 47E, 2975 (1993).

15. T. Kapitaniak, Chaotic Oscillations in Mechanical Systems, Manchester University Press, Manchester (1991).

16. K. Weickmann, J. Geophys. Res., 963187 (1991)

J.P. Mc Creary and D.L. Andeson, J. Geophys. Res., 963125 (1991)

J. Brindley, T. Kapitaniak and A. Barcilon, Phys. Lett. 167A, 179 (1992)

17. E. Lorenz, J. Atmos. Sci., 20, 130 (1963); Tellus, 17, 321 (1965); WMO, GARP Pub. Ser., 16132 (1975)

18. T.N. Palmer, Bull. Amer. Meteorological Soc., 74, 49 (1993)

19. R. Madden and P.R. Julian, J. Atmos. Sci., 28, 702 (1971)

20. J. Brindley and T. Kapitaniak, in preparation. 